\title{
Attracting the interprofessional collaboration between physical therapy, speech therapy and ABSN nursing students working with patients diagnosed with stroke during simulation
}

\author{
Debra R. Wallace, Jaya Mini Gill \\ California State University of Northridge, Los Angeles, California, United States
}

Received: July 14, 2017

DOI: $10.5430 /$ jnep.v8n3p56
Accepted: October 31, 2017

Online Published: November 6, 2017

URL: https://doi.org/10.5430/jnep.v8n3p56

\begin{abstract}
The purpose of this mixed methods interprofessional simulation was to assess health science university students in physical therapy, speech therapy, and nursing to the positive role of interprofessional collaboration by means of a live actor stroke simulation. The interprofessional simulation was divided into two segments which was comprised of: 1) the application of various teaching methods and orientation to the simulation lab, and 2) taking part in the organized simulated interprofessional care plan and subsequently participating in the debriefing and self-reflective exercises learning experiences. Logistic regression was used to measure quantitative outcomes including the Simulation Evaluation Survey questionnaire. The results were statistically significant. Qualitative data was obtained during simulation debriefing sessions, and was coded and analyzed. Incorporating the importance of inter-professional collaboration in professional program students helps promote team work, leadership, problem solving, critical thinking, and communication. The authors recommend incorporating interprofessional simulation in the curriculum for health care educational programs.
\end{abstract}

Key Words: Interprofessional collaboration, Clinical practice, Speech therapy, Physical therapy, Nursing

\section{INTRODUCTION}

For health care professionals, the importance of integrating teamwork and communication is vital to providing effective patient care. As newly graduate students, it is important for one to become proficient at their skills and provide safe and effective care regardless of the increasingly responsibilities. New graduates must master their professional skills despite increasing obligations, responsibilities and challenges. Stroke, is a common health condition among patients admitted to the emergency room. The World Health Organization
(WHO) defines health as an examination of "the complete physical, mental and social wellbeing and not just the absence of disease or illness". ${ }^{[1]}$ A study by Dondorf, Fabus and Ghassemi (2016) demonstrates how the perspectives and actions of nurses are changed with interprofessional collaboration amongst nurses and speech therapists when working the patient population diagnosed with dysphagia. ${ }^{[2]}$ Interprofessional collaboration is a journey of self-discovery that leads to better practitioners. ${ }^{[3]}$ One study revealed an increase in student's self-awareness following the application

\footnotetext{
*Correspondence: Debra R. Wallace; Email: Debra.Wallace@ @sun.edu; Address: California State University of Northridge, Los Angeles, California, United States.
} 
of reflective practice. ${ }^{[4]}$ By nurses reflecting with colleagues throughout the day, it showed how this support increased their confidence in their decision-making process.

Xia, Wu and Cheng (2017) stated that interprofessional collaboration is imperative to increase patient outcomes in the hospital. ${ }^{[5]}$ A contributing factor included how new graduates interacted with their preceptors and the professional environment to attain a sharper image of their own performance. Interprofessional collaboration can be used to bridge the theory-practice gap by encouraging examination, exploration and connections. ${ }^{[6]}$ Concerns presented regarding medical errors and health care quality along with team-based care to address such concerns are shown (see Table 1).

\subsection{Aims}

The purpose of this study was to assess the relationship demonstrated through interprofessional collaboration in 295
Accelerated Bachelors of Science Nursing (ABSN) students using QSEN based rubrics. Factors discussed include how nursing students interact with other health care disciplines and build on their knowledge formed during their clinical rotation. Responses were recorded on a likert scale $(1=$ completely disagree and $5=$ completely agree).

\subsection{Literature review}

Interprofessional education is a collaborative approach to develop healthcare students as future interprofessional team members and a recommendation suggested by the Institute of Medicine. ${ }^{[8]}$ Complex nursing issues can be best addressed by interprofessional teams. Training future healthcare providers to work in such teams will help facilitate this model resulting in improved healthcare outcomes for patients.

Table 1. Interprofessional collaboration clinical significance

\section{INTERPROFESIONAL COLLABORATION CLINICAL SIGNIFICANCE ${ }^{[7]}$}

- National reports on healthcare quality and safety led to recommendations to change fundamentally how healthcare professionals are educated to deliver care.

- Core competencies for interprofessional team-based care help guide educational change from pre-licensure to continuing education.

- "Collaborating Across Borders" conferences synergize interprofessional education developments in the United States and Canada. - A new Institute of Medicine Forum is linking educational changes to health professions' workforce issues.

In a study by Dondorf, Fabus, and Ghassemi (2015) emphasis was placed on interprofessional team building skills, knowledge of professions, patient centered care, service learning, the impact of culture on healthcare delivery and an interprofessional clinical component. ${ }^{[2]}$ The community-based experience demonstrates how interprofessional collaborations provide service to patients and how the environment and availability of resources impact one's health status.

\subsection{Teamwork between speech, physical therapists and nurses}

The partnership between physical therapy, speech therapy and nursing is necessary in the assessment, planning, implementation, and evaluation of stroke in the hospital setting. ${ }^{[9]}$ Nursing assessment of a stroke patient is imperative and requires full understanding of all elements. Nurses provide the around-the-clock observation and monitoring of patients in the acute care setting. Having direct open communication by a delivery team of nurses and physical therapists can improve the quality of overall patient care in individuals with a swallowing disorder. As an example, if a patient is exhibiting signs of coughing and state they are unable to swallow liquids, a nurse is able to relay this information to the Speech Language Pathologist (SLP). SLP's primary role is to evaluate and treat individuals with swallowing disorders. The patient can then be re-assessed and appropriate precautions can be taken to improve safety. According to the WHO, collaboration amongst disciplines allows for improved management of health care services, thus resulting in better outcomes and enhanced patient safety. ${ }^{[10]}$ Additionally, with multidiscipline collaboration, the overall goal is to minimize a patient's length of stay, avoid medical complications, and reduce mortality rates. Amongst caregivers, this would also lead to reduced staff turnover, tensions, and conflict. ${ }^{[1,12]}$ The primary focus of this article is collaboration between different team members to promote patient safety. ${ }^{[13]}$ As stated earlier, stroke can have a profound effect on patients, their families, and lead to numerous medical issues, or even death. There are improved outcomes when there is open communication between multi-disciplinary teams for patients are treated in a stroke unit.

\subsection{The assessment of a patient with stroke and the role of nursing}

The management of stroke is a key process for nurses care of patients. The National Institute of Health Stroke Scale 
(NIHSS) was compared to the Nursing Admission Dysphagia Screening Tool and was found to be more suitable in predicting dysphagia. The use of NIHSS clinical stroke assessment has been used widely in stroke patients as an early screening tool for dysphagia as it has been found to be a valid for predictor for predicting lesion size, stroke severity, and long term and short term outcomes. ${ }^{[14]}$ Bravata et al. (2009) showed that two screening assessments for stroke can be used by nurses which include the NIHSS and/or Nursing Admission Dysphagia Screening Tool, and an SLP consultation, which has been known to be the gold standard. ${ }^{[15]}$ It is critical for patients with swallowing difficulties to be assessed with a formal dysphagia screening protocol within 24 hours of the initial admission. It is also imperative that nurses are educated on performing dysphagia screening tools to appropriately implement strategies that will allow for optimal patient care. ${ }^{[16]}$ The advantages in providing training to nurses for screening patients with dysphagia has been studied by many researchers. ${ }^{[17]}$ In some facilities, an initial screen that prompts for a further dysphagia assessment by a speech therapist is made by the nurse. There are multiple protocols available created by different researchers for conducting a dysphagia screen. Therefore, since there is no universal screen, different facilities are either utilizing different screening tools or not using a screening tool at all. In one study, it was found that nurses who were trained by speech therapists to triage patients at risk for dysphagia using a tool generated by investigators, were appropriately trained on the signs and symptoms for possible dysphagia or aspiration as training is critical for successful screening. ${ }^{[18]}$ When these results are recorded into the computer system, it alerts for a speech therapist consult for further evaluation. By working as a team, one is able to effectively communicate with other disciplines and provide improved quality of care. ${ }^{[19]}$ With a lack of clinical education and simple nursing interventions in dysphagia, nursing staff members may be hesitant to perform such screening. ${ }^{[20,21]}$ A study by McHale et. al (1998), discusses the use of dysphagia practice guidelines and expert nursing knowledge in the treatment of stroke patients, as well the lack of material present in nursing textbooks regarding the responsibility as nurses in the assessment and management of stroke. ${ }^{[22,23]}$ This is where an interprofessional collaboration with different entities can improve patient care. The WHO (World Health Organization) proposes some methods by which health care professionals may interact as with one another to transform the way care is delivered. For instance, interdisciplinary education, including dysphagia workshops, in-services, and individual instruction should be evidence-based to provide staff with enhanced knowledge in caring for patients with dysphagia.
The WHO provides examples of approaches which may be implemented to provide individualized care. This will also outline interprofessional education to include staff training, institutional support, and learning outcomes. ${ }^{[24]}$ Partnership for stroke with both professionals should promote a collaborative approach with the entire team, thus reducing the risk of complications, providing a managed care environment, and developing focused outcomes to help provide effective care. ${ }^{[25]}$

\subsection{The treatment process of stroke and the nursing workforce}

Identifying individuals with stroke early and implementing proper interventions may improve stroke outcome and improve quality of life for individuals with stroke. ${ }^{[26]}$ Nurses historically have been in the foreground of hospital improvement programs. In acute care settings, nurses play a pivotal role and tend to be the first to identify stroke, and characterize individuals with stroke and begin interventions to prevent further complications. Collaboration involving both nurses and speech therapists should appear throughout the care continuum when caring for patients presenting with dysphagia. ${ }^{[27,28]}$ Speech therapists create a treatment plan accordingly to their assessment and evaluation measures. Following a dysphagia evaluation, the physical therapist and speech therapist will transmit the results of their assessments, recommendations, and interventions to the nurse. By implementing interprofessional learning in nursing education, it will allow one to improve overall health outcomes, and provide the best client-centered and quality of care. ${ }^{[29]}$

\subsection{Interprofessional simulation analysis}

Interprofessional teamwork leads to improvement in multiple areas such as coordination and collaboration, and helps to deliver patient-centered care and increase patient satisfaction. In our modern healthcare system, this team-based care is shown to be at the forefront. The Interprofessional Education Collaborative (IPEC) report of 2011 laid out the concept of interprofessional collaborative practice consisting of three competencies. ${ }^{[30]}$ The competencies of communication and teamwork can be seen in the following case scenario of two Registered Nurses (RNs) being the main caregivers for a patient. In an acute care environment, nurses and physicians work together in collaborative care. In this case, the team provided effective communication between the patient and her family. This in turn, allowed the family to have an increased understanding of the patient's state of health and have an agreement with the RNs' education. Although physicians knew the chance of a complete cure was slim, the family did not realize this. Nurses the ones to spend the most time with patients and are seen to be the eyes and ears of physi- 
cians. Therefore, physicians must appreciate the value of a nurses' opinions and suggestions, as nurses are the ones to provide direct care of patients. ${ }^{[31]}$ A doctor's responsibility is to provide the patient and their family the diagnosis, prognosis, and treatment options. As a team leader, the doctor is responsible for establishing clear guidance and vision for the team, while listening to and offering support and supervision to team members. With the third competency being staffing, sufficient staffing is required to allow nurses to meet patients' needs. ${ }^{[32]}$ This allows for a positive impact on patient outcomes as nurses need a proper combination of skills and competencies to care for patients. ${ }^{[33]}$ A multidisciplinary team also allows for a positive influence on patients' medical management and medication counseling. ${ }^{[34]}$

\section{MeTHODS}

\subsection{Setting and participants}

Encompassing interprofessional collaboration within the simulation training was accepted as being a part of a component in nursing education consisting of structured aims and outcomes. Participation in the interprofessional collaboration simulation is part of a mandatory curriculum of students in the ABSN program prior to partaking their medical surgical clinical rotation. Each simulation consisted of three students to a group with and scenarios running three times daily. Prior to starting the scenario, nursing, physical therapy, and speech therapy students were permitted to bring notes, pens, and assessment tools. Students were provided with a short briefing and given physician and nursing notes, along with any pertinent laboratory results. Any necessary resources were made available during the scenario as requested. During the simulation, the manikin was pre-programmed according to the case scenario. Scenarios also involved the implementation of any required interventions such as insertion of a peripheral intravenous catheter or an indwelling urinary catheter. Interdisciplinary groups consisting of nursing, physical therapy and speech therapy students working as a team were given 30 minutes to perform an evaluation of the client. Roles defined within the scenario included nursing students acting as caregivers to provide the initial contact with the patient and family members, and later performing a focused physical assessment. Physical therapy and speech therapy students acted in a consulting role by evaluating the patient using assessment tools focusing on physical, neurological, and cognitive testing.

During the simulation, nursing students portraying the roles of a patient and family member were encouraged to be involved in their care and ask their interprofessional healthcare team consisting of nursing, physical therapy, and speech therapy students questions pertaining to their treatment plan, re-

Published by Sciedu Press covery of function, quality of life, and discharge. All student groups being novice practitioners were able to communicate with each other, offer support, and answer the questions according to their expertise which were proposed by patients and their family members. With this collaboration model amongst the different disciplines came the requirement to meet a patient's needs. Physical therapy students participated in the scenario with nursing and speech therapy students by assessing a patient's functional needs and the interventions needed to provide safe patient care. This teamwork between the different professions allowed for one to participate as a unit during this problem-solving phase.$^{[35]}$ For example, nurses intervened in the simulation by providing vital signs and overall patient status; whereas physical therapy provided insight on to safely and properly mobilize such patient population. These interventions between the different groups were discussed to provide the optimal care to patients. At the end of the simulation, a structured debriefing session followed where students answered questions pertaining to the following: (1) What approach could the nurse have taken if there was something concerning? (2) What was the first indication that a speech therapist may be needed? What might that action have been done by the physical therapist? Was there anything else alarming? Physiologically, describe what was occurring. (3) Was there anything that took you by disbelief in learning about each other's role?

This questionnaire encouraged students to answer such questions about the simulation scenario. During the debriefing process, it was imperative for all members participating in the simulation and acting as observers to use positive communication to prevent embarrassment on any member playing a role in the simulation. This allowed faculty to gain insight on how one approaches a clinical scenario.

\subsection{Data collection}

A checklist was developed by the authors by means of providing a case flow description of the expected actions during the simulation which a learner is expected to carry out. At the end of the simulation, an evaluation survey was designed to assess the relevance of simulation debriefing and evaluate whether this activity assists students in developing greater problem-solving skills, and clinical competencies. Face validity was established after board of experts previewed the rubric for its significance and transparency. Evaluating content validity is an estimate to measure whether an item is comprehensive and is adequately representative of the population or characteristics being measured. As for being consistent with the data collection, a clinical faculty member was responsible for scoring all student performances equivalent to a likert grading rubric for scaling responses with answers 
ranging from 1 (strongly disagree) to 4 (strongly agree).

\subsection{Ethical consideration}

After approval from the Institutional Review Board of the University, the implementation of this study was to provide the foundation for establishing learning goals as a component of the program syllabus. Regarding ethical guidelines, the participation of subjects in the evaluation portion of the simulation was completely voluntary and would in no way alter their performance evaluation in the course. Questionnaires provided to students remained anonymous and confidential as all data was reported in aggregate.

\subsection{Data analysis}

To assess student satisfaction with the interprofessional collaboration simulation analysis of data was completed using the Statistical Package for the Social Sciences (SPSS) 22.0 for Windows. This assessment project used QSEN-based rubrics to evaluate interprofessional collaboration in ABSN nursing students. Interprofessional collaboration is important in helping students analyze their simulation performance. Using assessment data, this study showed that interprofessional collaboration is related to critical thinking, which is an essential component for safe nursing care. In this study, the expectations considered for logistic regression was comprised of sample size, multicollinearity, independence of errors, and outliers. According to Polit and Beck (2014), it was found that 10 subjects at minimum is required to adequately predicting logistic regression. A sample size of 295 subjects was used in this study; therefore, the model accurately reflects the five debriefing predictors. ${ }^{[30]}$

Spearman's nonparametric correlation, also known as Spearman's correlation $(r)$ test was used to predict the bivariate relationships between problem solving and the different components of interprofessional collaboration. It allows one to measure the strength association which exists between two variables. A bivariate association equivalent to or greater than .7 implicates multicollinearity. According to Table 2, confirmed the rationale and results show that all values were less than .7, implying that all five components of interprofessional collaboration and problem solving allow sufficient reflection on student's clinical judgement and approach to patient care, provide supportive and constructive feedback, give feedback that is helpful to learning, provide adequate time given to reflect and discuss clinical performance.

\section{RESUlts}

\subsection{Quantitative results}

Spearman's rho correlation coefficient showed significant relationship in the analysis between all components of interprofessional collaboration; and significant correlation in all components of problem solving, allowing reflection on students' clinical judgment ( $r=.24, p=.00)$; feedback supportive and constructive $(r=.24, p=.00)$; feedback helpful to learning $(r=.23, p=.00)$; adequate time to reflect and discuss clinical performance $(r=.22, p=.00)$; and helping understand action rationale $(r=.33, p=.00)$ thus a positive association was observed between simulation improving problem solving and all aspects of interprofessional collaboration (see Table 2). Of the 295 students, 95\% ( $\mathrm{N}=280)$ came to an understanding that simulation improved their critical thinking (see Table 3 ). The logistic multivariate regression model showed statistical significance in only 3 out of 5 interprofessional collaboration components predicted stronger problem-solving skills: allowing reflection on students' clinical judgment $\left(\chi^{2}=33.349, p=.01\right)$, adequate time to reflect and review clinical performance $\left(\chi^{2}=0.071\right.$, $p=.03$ ), and helping understand the rationale for the actions and responses to performances $\left(\chi^{2}=121.26, p=.00\right)$. Interprofessional collaboration as "supportive and constructive" and "feedback helpful to learning" showed no statistically significant relationship to the independent variable critical thinking (see Table 3).

Table 2. Bivarian correlation box

\begin{tabular}{|c|c|c|}
\hline Problem Solving & Correlation & p-value \\
\hline Interprofessional collaboration $\&$ reflection on problem solving $\&$ approach to patient care & .24 & $.00 *$ \\
\hline Interprofessional collaboration feedback was supportive \& helpful & .24 & $.000 *$ \\
\hline Interprofessional collaboration feedback helpful to learning & .23 & $.000 *$ \\
\hline Adequate collaboration time to discuss performance & .22 & $.00 *$ \\
\hline Interprofessional collaboration helped understand action rationale & .33 & $.000 *$ \\
\hline
\end{tabular}

\subsection{Qualitative results}

Recorded simulation debriefing sessions were transcribed and analyzed in search for recurrent themes. The resultant two themes embraced: The importance of interprofessional communication and teamwork and respecting the simulation learning experience on clinical judgement (see Table 4). 
Table 3. Significance, odds ratio, and ci for problem solving and interprofessional collaboration

\begin{tabular}{lllll}
\hline Problem Solving & \multirow{2}{*}{ Sig. } & \multirow{2}{*}{ Odds Ratio } & \multicolumn{2}{c}{ 95\% CI for Odds Ratio } \\
\cline { 3 - 5 } & & & Lower & Upper \\
\hline Interprofessional collaboration \& reflection on clinical judgment \& patient care & $.011^{*}$ & 33.349 & 2.245 & 522.437 \\
Interprofessional collaboration feedback was supportive \& constructive & .123 & .109 & .006 & 1.826 \\
Interprofessional collaboration feedback was helpful to learning & .220 & 5.965 & .343 & 103.708 \\
Adequate collaboration time to discuss performance & $.030^{*}$ & .071 & .006 & .769 \\
Interprofessional collaboration helped understand action rationale & $.001^{*}$ & 121.26 & 7.469 & $1,907.635$ \\
\hline${ }^{*} p \leq .05$ & & & &
\end{tabular}

Table 4. Qualitative results

- Importance of interprofessional communication and teamwork

- Respecting the simulation learning experience on clinical judgement

\section{IMPLICATIONS AND RECOMMENDATIONS}

\subsection{Study limitations}

The findings of the study were limited by the study's sample size and may not be generalizable to a larger group of nursing students. A single-institution review of 295 students was used for this study as a statistical method of convenience sampling and therefore limits the comparison to nonrandom sampling which may only be construed within that perspective. With a small sample size, results cannot be generalized to all nursing students; therefore, results need to be interpreted with caution. Additionally, a small sample size may also justify obtaining near significance results which signify complex cognitive competencies comprising of developing problem solving skills, and reflecting on clinical judgement and patient care. Consequently, potential quantitative and qualitative studies should be utilized with a greater number of samples to investigate if simulation is capable in enhancing these essential characteristics. More research amongst nursing students in various stages of the nursing curriculum and at different institutes would permit for a more systematic review examining the benefits of simulation and its relation to an interprofessional approach...

\subsection{Recommendations}

The integration of interprofessional collaboration in simulation models is recognized to be an important variable for clinical faculty to incorporate into nursing education. Working with professional members of different disciplines is an important factor in delivering collaborative, holistic, and patient-centered care as each comes with a specific set of competencies. ${ }^{[36]}$ "By changing the way health workers inter- act with one another, the culture of the working environment and attitudes of the workforce will change, improving the working experience of staff and benefiting the community as a whole." ${ }^{[37]}$ As discussed, the WHO guidelines provide speech therapists, physical therapists and nurses with approaches that may be implemented to strengthen collaboration between one another and help provide individuals with dysphagia the highest quality care. ${ }^{[37,38]}$

Interprofessional collaboration also allows for one to incorporate problem solving into the setting of learning by accepting different viewpoints and suggestions. This helps aid nursing students in enhancing their skills in competency and critical thinking. Additionally, by understanding the results presented from this study, it will allow one to gain a better perception of how incorporating the practice of simulation into education impacts an individual's critical thinking skills. Prospective research may aim to focus on funding larger demographics, analyzing quantitative data from surveys, and using reliable and validated tools.

\section{Bio Statement}

Debra Wallace, DNP, RN, FNP-BC, serves as part time faculty in the School of Nursing at California State University of Northridge and mentors nursing students at Cal State University of Northridge and nurse practitioner students while working for Kaiser Permanente in Santa Clarita, CA.

Dr. Jaya Gill earned her Bachlor of Science in Biology from UCLA in 2006. In 2011, she earned her MD degree from American University of the Caribbean, and in 2014 she earned a Bachelor of Science in Nursing degree from CSUN. She has worked as a critical care registered regisitered nurse and has gained invaluable exerience in acute care nursing.

\section{Conflicts of Interest Disclosure}

The authors declare that there is no conflict of interest. 


\section{REFERENCES}

[1] "health." World Health Organization. 2017. Available from: http: //www.who.int/about/mission/en/

[2] Dondorf K, Fabus R, Ghassemi AE. The interprofessional collaboration between nurses and speech-language pathologists working with patients diagnosed with dysphagia in skilled nursing facilities. Journal of Nursing Education and Practice. 2015; 6(4): 17-20. https://doi.org/10.5430/jnep.v6n4p17

[3] Bridges DR, Davidson RA, Odegard PS, et al. Interprofessional collaboration: three best practice models of interprofessional education Medical Education Online. 2011; 16. https : //doi .org/10.340 2/meo.v16i0.6035

[4] Bradbury-Jones C. Globalisation and its implications for health care and nursing practice. Nursing Standard. 2009; 23(25): 43-47. PMid:19323125 https://doi.org/10.7748/ns2009.02.23.2 $5.43 . c 6831$

[5] Xia L, Wu H, Cheng Y. Interprofessional collaboration strategies: A hematology unit case study. Journal of Nursing Education and Practice. 2017; 7(4): 51-54. https ://doi.org/10.5430/jnep.v $7 \mathrm{n} 4 \mathrm{p} 51$

[6] Hazelton JP, Orfe EC, et al. The impact of a multidisplinary safety ck list on adverse procedural events during bedside tracheostomy. The Journal of Trauma and Acute Care Surgery. 2015; 79(1): 111-6. PMid:26091323 https://doi.org/10.1097/TA.00000000000 00700

[7] Schmitt MH, Gilbert JHV, Brandt BF, et al. The Coming of Age for Interprofessional Education and Practice. The American Journal of Medicine. 2013; 124(4): 284-288. https ://doi.org/10.1016/j . amjmed.2012.10.015

[8] Institute of Medicine. The Future of Nursing: Leading Change, Advancing Health. Washington, DC: National Academies Press; 2011. Available from: http://www.aanp.org/practice/iom-futur e-of-nursing Accessed December 16, 2013.

[9] Jeffries PR. A framework for designing, implementing, and evaluating simulations used as teaching strategies in nursing. Nursing Education Perspective. 2005; 26(2): 96-103.

[10] World Health Organization. 2009. Available from: http://www.wh o.int/chp/steps/stroke/en/

[11] Stroke Unit Trialists' Collaboration. Organized inpatient (stroke unit) care for stroke. In the Cochrane Database of Systematic Reviews. 2007; (4): Art. PMid:17943737 https://doi.org/10.1002/14 651858. CD000197.pub2

[12] National Audit Office. Progress in improving stroke care. London, National Audit Office. 2010.

[13] Kilbride CB, Meyer JM, Flatley M, et al. Strokeunits: The implementation of a complex intervention. Educational Action Research Journal. 2005; 13: 479-503. https ://doi .org/10 .1080/147593 90500200341

[14] Department of Health. National stroke strategy. London: The Stationery Office. 2007.

[15] Bravata DM, Daggett VS, Woodward-Hagg H, et al. Comparison of two approaches to screen for dysphagia among acute ischemic stroke patients: nursing admission screening tool versus National Institutes of Health stroke scale. Journal of Rehabilitation Research and Development. 2009; 46(9): 1127-34. PMid:20437319 https://doi.org/10.1682/JRRD. 2008.12.0169

[16] Sandhaus S, Zalon ML, Valenti D, et al. Promoting evidencebased dysphagia assessment and management by nurses. Journal of Gerontology Nursing. 2009; 35(6): 20-7. PMid:19537291 https : //doi.org/10.3928/00989134-20090331-08

[17] Suter E, Arndt J, Arthur N, et al. Role understanding and effective communication as core competencies for collaborative practice. Jour- nal of Interprofessional Care. 2009; 23(1): 45-51. PMid:19142782 https://doi.org/10.1080/13561820802338579

[18] Hines S, Wallace K, Crowe L, et al. Identification and nursing management of dysphagia in individuals with acute neurological impairment (update). International Journal of Evidence Based Healthcare. 2011; 9(2): 148-150. PMid:21599843 https://doi .org/10.111 $1 / \mathrm{j} .1744-1609.2011 .00211 . \mathrm{x}$

[19] McCullough KC, Estes JL, McCullough GH, et al. RN compliance with SLP dysphagia recommendations in acute care. Topics in Geriatric Rehabilitation. 2007; 23(4): 330-340. https ://doi .org/10 .1097/01.TGR.0000299161.44869.26

[20] Kameg K, Howard VM, Clochesy J, et al. The Impact of High Fidelity Human Simulation on Self-Efficacy of Communication Skills. Issues in Mental Health Nursing. 2010; 31(5): 315-323. PMid:20394477 https://doi.org/10.3109/01612840903420331

[21] Curran GM, Sullivan G, Mendel P, et al. Implementation of the CALM intervention for anxiety disorders: a qualitative study. Implementation Science. 2012; 9(7): 1-11. https ://doi .org/10.118 6/1748-5908-7-14

[22] McHale JM, Phipps MA, Horvath K, et al. Expert nursing knowledge in the care of patients at risk of impaired swallowing. Journal of Nursing Scholarship. 1998; 30(2): 137-41. https ://doi.org/10 .1111/j.1547-5069.1998.tb01268.x

[23] Hinchey JA, Shephard T, Furie K, et al. Formal Dysphagia Screening Protocols Prevent Pneumonia. Stroke. 2005; 36: 1972-1976. PMid:16109909 https://doi.org/10.1161/01.STR.0000177 $529.86868 .8 \mathrm{~d}$

[24] World Health Organization. Constitution of the World Health Organization. 2006. Available from: http://www. who.int/governan ce/eb/who_constitution_en.pdf

[25] Kilbride C, Perry L, Flatley M, et al. Developing theory and practice: Creation of a Community of Practice through Action Research produced excellence in stroke care. Journal of Interprofessional Care. 2011; 25(2): 91-97. https://doi.org/10.3109/13561820. 201 0.483024

[26] Suter E, Arndt J, Arthur N, et al. Role understanding and effective communication as core competencies for collaborative practice. Journal of Interprofessional Care. 2009; 23(1): 45-51. PMid:19142782 https ://doi.org/10.1080/13561820802338579

[27] Fitzgerald L, Dopson S. Professional boundaries and the diffusion of innovation. In S. Dopson \& L. Fitzgerald (Eds.), Knowledge to action? Evidence-based health care in context. Oxford: Oxford University Press; 2006.

[28] Fitzgerald L, Dopson S, Ferlie E, et al. Knowledge in action. In S. Dopson, L. Fitzgerald (Eds.), Knowledge to action? Evidence-based healthcare in context. Oxford: Oxford University Press; 2006.

[29] Edmiaston J, Connor LT, Ford AL. SWALLOW-3D, a simple 2minute bedside screening test, detects dysphagia in acute stroke patients with high sensitivity when validated against video-fluoroscopy (abstract). Stroke. 2011; 42: e352.

[30] Interprofessional Education Collaborative Expert Panel. Core competencies for interprofessional collaborative practice: report of an expert panel. Washington, DC: Interprofessional Education Collaborative; 2011.

[31] Pillow M, ed. Improving Hand-off Communication. Oakbrook Terrace, IL: The Joint Commission; 2007.

[32] Agency for Healthcare Research and Quality. Team STEPPS 2.0 Essentials. Available from: http://www.ahrq.gov/professio nals/education/curriculum-tools/teamstepps/instru ctor/essentials/slessentials.html\#s5 Accessed July 20, 2015. 
[33] Begley CM. Developing inter-professional learning: tactics, teamwork and talk. Nurse Education Today. 2009; 29(3): 276-283. PMid:19124176 https://doi.org/10.1016/j.nedt.2008.09 .006

[34] Marshall S, Harrison J, Flanagan B. The teaching of a structured tool improves the clarity and content of interprofessional clinical communication. Qual Safe Health Care. 2009; 18(2): 137-140. PMid:19342529 https://doi.org/10.1136/qshc. 2007.0252 47

[35] Shrader S, Dunn B, Blake E, et al. Incorporating Standardized Colleague Simulations in a Clinical Assessment Course and Evaluating the Impact on Interprofessional Communication. Am J Pharm Educ. 2015; 79(4): Article 57.

[36] Polit DF, Beck CT. Nursing Research: Generating and Assessing Evidence for Nursing Practice. 9th ed. Philadelphia, PA: Lippincott Williams \& Wilkins; 2014.

[37] Cox M, Naylor M. Transforming patient care: aligning interprofessional education with clinical practice redesign. Conference recommendations. Atlanta, GA; 2013.

[38] World Health Organization. Framework for action on interprofessional education and collaborative practice. Geneva: WHO; 2010. 